\title{
A Coverage Area-Based Cooperation Technique for SWIPT-Enabled Systems with Mobility
}

\author{
Yuan Guo, Christodoulos Skouroumounis, and Ioannis Krikidis \\ IRIDA Research Centre for Communication Technologies \\ Department of Electrical and Computer Engineering, University of Cyprus \\ Email: \{yguo0001, cskour03, krikidis\}@ucy.ac.cy
}

\begin{abstract}
We investigate the performance of the simultaneous wireless information and power transfer (SWIPT)-enabled mobile networks, based on a novel coverage area-based coordinated multipoint transmission (CA-CoMP) technique. In particular, the proposed scheme exploits the cooperation technique by appropriately defining the set of cooperative base stations, aiming at reducing the handover rate of the SWIPT-enabled battery-operated mobile users (MUs). Using stochastic geometry tools, we first establish an analytical and tractable framework to investigate the achieved information decoding and energy harvesting performance of the proposed scheme, in terms of the coverage probability, the average spectral efficiency and the average harvested energy. Moreover, analytical expressions for the inter- and intra-cell handover rates are derived. Our results indicate the ability of our proposed scheme to significantly reduce the handover rate of MUs. Finally, simulation results verify that the CA-CoMP scheme achieves a higher average spectral efficiency and a higher average harvested energy compared with the conventional cooperative technique.

Index Terms-Cooperation, SWIPT, handover, stochastic geometry.
\end{abstract}

\section{INTRODUCTION}

Simultaneous wireless information and power transfer (SWIPT) is a promising communication paradigm, where wireless devices extract both information and energy from the received radio-frequency (RF) signals [1]. Due to the practical limitations, a receiver cannot decode information and harvest energy independently from the same signal without losses. Fortunately, the concept of SWIPT becomes feasible by splitting the received RF signals in two parts; one part is used for information transfer and another part is used for power transfer. The partitioning of the RF signal can be performed either in the time, the power, or the space domain [1] [2].

The SWIPT technology in the context of large-scale networks has been widely-investigated in the literature. In [2], the authors investigated the performance limits of emerging wireless-powered communication networks, revealing the fundamental data-energy tradeoff in designing multiple-input multiple-output (MIMO) systems for maximising the efficiency of SWIPT technique. In the context of cellular networks,

This work has received funding from the European Research Council (ERC) under the European Union's Horizon 2020 research and innovation programme (Grant agreement No. 819819) and from the Marie SkłodowskaCurie project PAINLESS under the European Union's Horizon 2020 research and innovation programme (Grant agreement No. 812991). This work was also co-funded by the European Regional Development Fund and the Republic of Cyprus through the Research and Innovation Foundation, under the project INFRASTRUCTURES/1216/0017 (IRIDA). the authors in [3] and [4] studied the simultaneously joint harvested energy and data transmission performance under time switching (TS) and power splitting (PS) schemes from the macroscopic point-of-view; the optimal partitioning parameters to achieve maximum joint energy harvesting (EH) and information decoding (ID) performance were demonstrated.

The idea of coordinated multi-point (CoMP) transmission has been regarded as a prominent strategy to significantly enhance link reliability of mobile networks [5] [6]. On the one hand, the end-user service quality can be greatly improved with the CoMP technique, by jointly processing signals which are received from multiple base stations (BSs) [7]. On the other hand, the CoMP transmission scheme generally results in more frequently connections altering with multiple serving BSs during mobile users' (MUs') motion [5]. In particular, the work in [5] investigated the concept of handover in usercentric cooperative wireless networks, where the handover rate under cooperation was derived and the optimal cluster size was theoretically quantified to maximize the date rate. In order to reduce the handover cost of the MUs, the authors in [6] proposed two movement-aware CoMP handover schemes, where the MUs association policy was designed based on the cell dwell-time. In addition to the inter-cell handover discussed in the aforementioned works, the authors in [8] investigated the intra-cell handover in 5G mobile networks (i.e., beam reselection), where the closed-form expression of the beam reselection rate was analytically derived.

In this paper, we study the performance of the SWIPTenabled battery-operated MUs, which are randomly moving within the network. Aiming to alleviate the MUs' handover rate and, therefore, to enhance the SWIPT performance (i.e., ID and $\mathrm{EH}$ ), we propose a novel coverage area-based CoMP (CACoMP) scheme. In particular, an appropriate coverage area threshold is initially defined, and then the MUs jointly decode information and harvest energy from multiple BSs, whose coverage area is greater than the pre-defined area threshold. We consider both inter- and intra-cell handovers for the MUs, where closed-form expressions of both the inter- and intracell handover rates are derived. Furthermore, using stochastic geometry tools, we derive the analytical expressions for the coverage probability, the average spectral efficiency as well as the average harvested energy of the MUs. Our results show that, by appropriately selecting the set of cooperative BSs, both inter- and intra-cell handover rates of MUs are significantly 
reduced. Finally, it is demonstrated that, compared to the conventional cooperative technique, the proposed CA-CoMP scheme enables a higher average spectral efficiency and a higher average harvested energy.

Notation: $\mathbb{R}^{d}$ denotes the $d$ dimensional Euclidean space; $\|x\|$ denotes the Euclidean norm of $x \in \mathbb{R}^{d} ; \mathbb{P}[X]$ denotes the probability of the event $X$ and $\mathbb{E}[X]$ represents the expected value of $X ; \Gamma(\cdot)$ and $\Gamma(\cdot, \cdot)$ denote the complete and the upper incomplete functions, respectively; ${ }_{2} F_{1}(\cdot, \cdot ; \cdot ; \cdot)$ is the Gauss hypergeometric function; and $\mathcal{G}[a, b]$ denotes the Gamma distribution with a shape and a scale parameter $a$ and $b$, respectively.

\section{SySTEM MODEL}

\section{A. Network and Channel Model}

We consider a single tier cellular network, where the BSs are spatially distributed based on a homogeneous Poisson point process (PPP) $\Phi=\left\{x_{i} \in \mathbb{R}^{2}\right\}$, with density $\lambda$, where $x_{i}$ denotes the spatial coordinate of the $i$-th BS. We assume that all BSs transmit signals with identical power $P_{t}$ [9]. Moreover, we assume that the MUs are uniformly distributed with a density $\lambda_{u} \gg \lambda$. In addition, we assume that each BS serves only one MU at a time per resource block, while each MU communicates with $N$ serving BSs based on our proposed CA-CoMP scheme introduced in Section III. Based on the Slivnyak's theorem and without loss of generality, we perform our analysis from the perspective of the typical MU, which is located at the origin [9].

All wireless signals are assumed to experience both largescale path loss effects and small-scale fading. Specifically, the small-scale fading is modeled as Rayleigh fading, where different links are assumed to be independent and identically distributed. Hence, the channel power gain is an exponential random variable with unit mean, i.e. $h \sim \exp (1)$ [3]. Moreover, the large-scale path loss between a transmitter at $X$ and a receiver at $Y$, is modelled by an unbounded singular path loss model, i.e. $L(X, Y)=\|X-Y\|^{\alpha}$, where $\alpha>2$ is the path loss exponent.

\section{B. Beamforming Model}

We assume that all BSs are equipped with multiple antennas, while all MUs have single omnidirectional antenna. We consider that each BS has a codebook of $2^{n}$ beamforming vectors with $n \in \mathbb{N}$, where the patterns of these beamforming vectors have non-overlapping main-lobes designed to cover the full angular range, i.e. $[0,2 \pi)$ [8]. Hence, the beamwidth for a BS is considered as the central angle of a sector, i.e. $\psi_{n}=\pi / 2^{n-1}$. Moreover, we consider a simple antenna pattern model where the main lobe is restricted to the beamwidth, thus the antenna gain of the link between a BS and a MU is given by $G=\left\{G_{m}, G_{s}\right\}$, with the corresponding probabilities $p_{G}=\left\{\frac{\psi_{n}}{2 \pi}, \frac{2 \pi-\psi_{n}}{2 \pi}\right\}$, where $G_{m}$ and $G_{s}$ represent the main and side lobe antenna gains, respectively [8]. Furthermore, we assume that perfect beam alignment can be achieved between a MU and its serving BSs by using the estimated angles of arrival, resulting in an antenna gain of $G_{m}$ [3].

\section{Mobility Induced Handovers}

We consider that the mobility of a MU within the network area is based on the random waypoint mobility (RWP) model, where each MU has arbitrary movement in the network with velocity $v \in\left[0, v_{\max }\right]$ [10]. During the motion of MUs, both inter- and intra-cell handovers may occur. Initially, inter-cell handover refers to the BSs handover that occurs, when the received signal strength of the target BSs exceeds that of the serving BSs; MUs establish connections with new serving BSs to maintain the best connectivity throughout the trajectory [5]. Moreover, the intra-cell handover refers to the process that is triggered when a MU moves out the one beam area of a BS, while a new beam has to be reselected based on the MU's current location (also referred to the beam reselection process) [8].

\section{SWIPT-enabled CoMP Technique}

All MUs are assumed to be capable of decoding information and harvesting energy from multiple BSs simultaneously, via TS or PS policies. More specifically, by adopting the TS policy, a MU spends a fraction $\tau \in[0,1]$ of the allocated time slot to decode the received signal, while for the rest of the time, it harvests energy. Analogously, for a PS-based MU's receiver, the received power is divided into two parts with a splitting ratio $\rho \in[0,1]$, where a fraction $\rho$ of the received power is consumed for the ID purpose, while the remaining power is inputted to the EH circuit [1].

\section{Coverage AREA-BASED COMP SCHEME AND HANDOVER ANALYSIS}

Our proposed CA-CoMP scheme targets the alleviation of the frequent handover process by the MUs in the considered CoMP-enabled mobile network, thereby improving the network throughput as well as enhancing the MUs' energy harvesting performance. Analytical and closed-form expressions for both the inter- and intra-cell handover process experienced by the MUs are investigated.

\section{A. Coverage Area-based CoMP Scheme}

The proposed CA-CoMP scheme is based on a two-level process. In the first-level phase, a set of the candidate BSs is determined based on the cell coverage area. Specifically, the BSs with a coverage area greater than a pre-defined area threshold $\mathcal{A}$, are selected into the candidate BSs set $\tilde{\Phi}$, i.e. $\tilde{\Phi}=\left\{x_{i} \mid \forall x_{i} \in \Phi, \mathcal{A}_{i}>\mathcal{A}\right\}$, where $\mathcal{A}_{i}$ is the coverage area of the BS located at $x_{i}$. Then, in the second-level phase, the MU selects from the set of candidate BSs, its $N$ closest BSs; the rest of the candidate BSs are ignored. Hence, the final set of the serving BSs is formulated as $\mathcal{C}=\left\{x_{1}, \cdots, x_{N}\right\}$, where $x_{i} \in \tilde{\Phi}$, denotes the location of the $i$-th closest serving BS of the MU.

It is worth mentioning that, the proposed CA-CoMP scheme is a flexible technique and according to the MUs' mobility within the network, the area threshold can be appropriately adjusted. In particular, for the high velocity MUs, i.e. $v \rightarrow \infty$, the handover overhead is the dominant factor that undermines 
the network performance. Therefore, a large area threshold is beneficial to adopt in order to greatly reduce MUs' handover rate. On the other hand, for a MU with low velocity or a static $\mathrm{MU}$, i.e. $v \rightarrow 0$, the handover overhead is negligible, and hence, a small area threshold is beneficial to keep MUs associate with the closest BSs to achieve the highest received signal strength.

\section{B. Inter-cell Handover Rate}

In this section, we investigate the average inter-cell handover rate of the MUs based on the CA-CoMP scheme. Since the serving BSs of a MU are selected from the set of candidate BSs, we first clarify the spatial distribution of the candidate BSs, i.e. $\tilde{\Phi}$. Let $p_{a}$ denote the probability that the coverage area of a BS is larger than the pre-defined threshold $\mathcal{A}$. Since the original spatial distribution of the BSs follows a homogeneous PPP, and based on the thinning property, the distribution of the candidate BSs is still uniform, with a density $\tilde{\lambda}=\lambda p_{a}$ [9]. In addition, the area of a Voronoi cell $\mathcal{A}_{i}$ can be accurately approximated by a Gamma distribution with a shape and a scale parameter $K$ and $K \lambda$, respectively, i.e. $\mathcal{A}_{i} \sim \mathcal{G}[K, K \lambda]$, where $K=3.5$ [11]. Hence, $p_{a}$ can be derived as follows,

$$
p_{a}=\mathbb{P}\left[\mathcal{A}_{i} \geq \mathcal{A}\right]=\frac{\Gamma(K, \mathcal{A} K \lambda)}{\Gamma(K)} .
$$

Based on the above, by associating a MU with its $N$ closest BSs from the set of the candidate BSs $\tilde{\Phi}$, the Euclidean plane $\mathbb{R}^{2}$ is separated into regions, forming an $N$-th order Voronoi tessellation [12]. Therefore, the handover of a MU is triggered when it crosses the boundaries of an $N$-th order Voronoi cell, where the average handover rate of a MU with a velocity $v$ is given by [5]

$$
\Xi_{c}=\frac{8 v \sqrt{\tilde{\lambda}} \Gamma(0.5+N)}{\pi^{3 / 2} \Gamma(N)} .
$$

\section{Intra-cell Handover Rate}

We now investigate the intra-cell handover of the MUs. Specifically, an intra-cell handover is triggered when a MU crosses the beam boundaries of the serving BSs, where a new beam has to be reselected for the downlink transmission. Since, a MU is jointly served by $N$ serving BSs, the intra-cell handover rate equals to the average number of beam reseletion with respect to $N$ serving BSs per unit time. Hence, based on the proposed CA-CoMP scheme, the intra-cell handover rate of the MUs is evaluated in the following theorem.

Theorem 1. Based on the proposed CA-CoMP scheme, the intra-cell handover rate of the MUs is given by

$$
\Xi_{b}=\frac{2^{n+1} v \sqrt{\tilde{\lambda}} \Gamma(0.5+N)}{\pi^{3 / 2} \Gamma(N)},
$$

where $v$ and $N$ is the velocity and the number of the serving $B S s$ of a $M U$, respectively.

Proof. See Appendix A.

\section{Network Performance}

In this section, we evaluate some key performance metrics of the SWIPT-enabled mobile cellular networks in the context of our proposed CA-CoMP scheme. More specifically, we first investigate the ID performance by calculating the coverage probability as well as the average spectral efficiency. Subsequently, we study the energy harvesting ability of the MUs by evaluating the mean level of the MUs' harvested energy per unit time.

\section{A. Coverage Probability}

An important performance metric for the considered wireless networks is the coverage probability. More specifically, by assuming that the serving BSs employ the non-coherent joint transmission policy [5], the downlink coverage probability is defined as the probability that a MU is able to achieve a certain SINR threshold $\beta$, i.e.

$$
\mathcal{P}_{c}(\beta)=\mathbb{P}\left[\frac{\left|\sum_{x_{i} \in \mathcal{C}} P_{t}^{1 / 2} r_{i}^{-\alpha / 2} h_{i}^{1 / 2}\right|^{2}}{\sum_{x_{i} \in \Phi \backslash \mathcal{C}} P_{t} h_{i} r_{i}^{-\alpha}+\sigma_{C}^{2}+\sigma_{N}^{2}} \geq \beta\right],
$$

where $r_{i}=\left\|x_{i}\right\|$ is the distance from the $\mathrm{BS}_{i}$ to the typical MU, $\sigma_{N}^{2}$ is the thermal noise, $\sigma_{C}^{2}=\sigma_{\text {cov }}^{2}$ and $\sigma_{C}^{2}=\sigma_{\text {cov }}^{2} /(1-$ $\rho$ ) for the TS and PS scheme, respectively, where $\sigma_{c o v}^{2}$ is a constant that accounts for the noise of the signal conversion from RF to baseband [3]. The analytical expression for the coverage probability is derived in the following theorem.

Theorem 2. Based on the proposed CA-CoMP scheme, the downlink coverage probability is given by

$$
\begin{aligned}
\mathcal{P}_{c}(\beta)= & \int_{0}^{\infty} \int_{0}^{r_{N}} \cdots \int_{0}^{r_{2}} \mathcal{L}_{I}(s) \exp \left(-\left(\sigma_{C}^{2}+\sigma_{N}^{2}\right) s\right) \\
& \times f_{r}\left(r_{1}, \ldots, r_{N}\right) \mathrm{d} r_{1}, \ldots, r_{N-1}, r_{N},
\end{aligned}
$$

where $\mathcal{L}_{I}(s)$ is the Laplace transform of the aggregate interference function, which is given by (5), $s=\beta /\left(G_{m} P_{t}\left(r_{1}^{-\alpha}+\right.\right.$ $\left.\left.\cdots+r_{N}^{-\alpha}\right)\right)$, and $f_{r}\left(r_{1}, \ldots, r_{N}\right)$ is the joint distribution of the distance to $N$ serving $B S s$, which is given by [11]

$$
f_{r}\left(r_{1}, \ldots, r_{N}\right)=(2 \tilde{\lambda} \pi)^{N} \exp \left(-\tilde{\lambda} \pi r_{N}^{2}\right) r_{1} \ldots r_{N}
$$

Proof. See Appendix B.

\section{B. Average Spectral Efficiency}

In order to further assess the ID performance of the MUs, we now investigate the average spectral efficiency (ASE) achieved by our proposed CA-CoMP scheme. In particular, the ASE refers to the average data rate achieved by a MU per unit bandwidth, which is given by [8]

$$
\mathrm{ASE}=T_{e}^{\mathrm{ID}} \int_{0}^{\infty} \frac{\mathcal{P}_{c}(z)}{z+1} \mathrm{~d} z
$$

where $\mathcal{P}_{c}(\cdot)$ is the coverage probability of MUs given in Theorem 2. Based on the selected SWIPT policy, $T_{e}^{\mathrm{ID}}$ is given by

$$
T_{e}^{\mathrm{ID}}= \begin{cases}\max \left\{0, \tau\left(1-T_{b} \Xi_{b}-T_{c} \Xi_{c}\right)\right\} & \text { TS policy } \\ \max \left\{0,1-T_{b} \Xi_{b}-T_{c} \Xi_{c}\right\} & \text { PS policy, }\end{cases}
$$

where $\tau$ is the time switching factor, $T_{b}$ and $T_{c}$ denote the signalling overhead delay of intra- and inter-cell handovers, respectively, $\Xi_{c}$ and $\Xi_{b}$ depict the inter- and intra cell handover rate, which are given by (1) and (2), respectively. 


$$
\mathcal{L}_{I}(s)=\exp \left(\sum_{G} 2 \pi \lambda r_{N}^{2} p_{G}\left(\frac{\left(p_{a}-1\right)_{2} F_{1}\left[1, \frac{2}{\alpha} ; \frac{\alpha+2}{\alpha} ;-\left(G P_{t} r_{N}^{-\alpha} s\right)^{-1}\right]}{2}+\frac{G P_{t} s r_{N}^{-\alpha}{ }_{2} F_{1}\left[1, \frac{\alpha-2}{\alpha} ; 2-\frac{2}{\alpha} ;-G P_{t} r_{N}^{-\alpha} s\right]}{\alpha-2}\right)\right)
$$

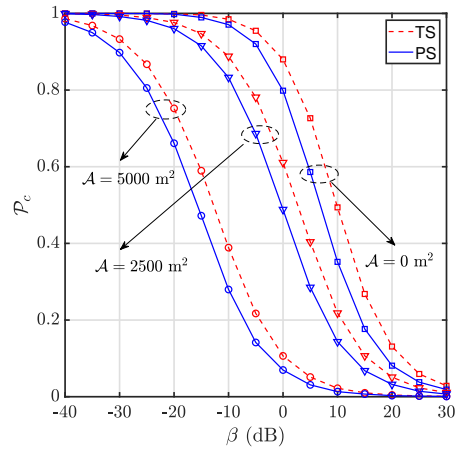

Fig. 1: Coverage probability versus $\beta$ for different area threshold $\mathcal{A} \in$ $\{0,2500,5000\} \mathrm{m}^{2}$.

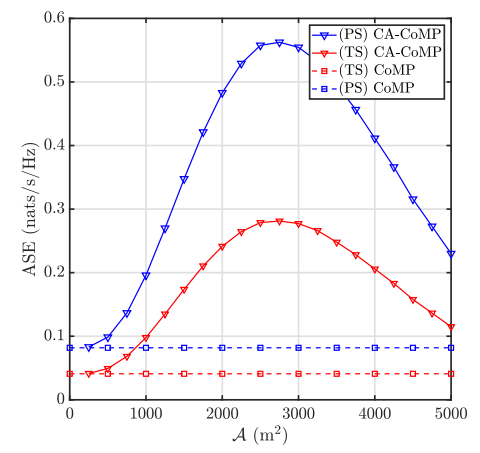

Fig. 2: Average spectral efficiency versus the area threshold $\mathcal{A}$ for TS and PS policies.

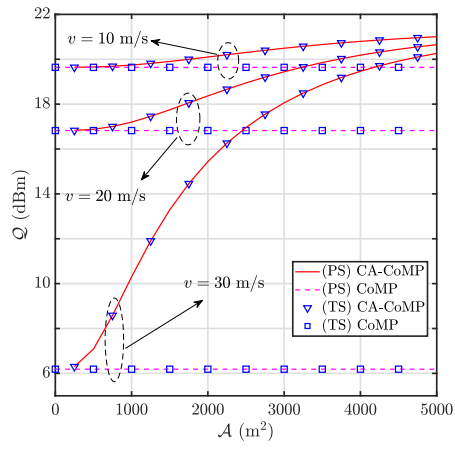

Fig. 3: Average harvested energy versus the area threshold $\mathcal{A}$ for TS and PS policies.

\section{Average Harvested Energy}

In this section we study the energy harvesting capability of the MUs. For the sake of simplicity, a linear energy harvesting model is considered to provide a general insight on the network performance [1] [3]. Hence, by considering both TS and PS policies, we derive the analytical expressions for a MU's average harvested energy in the following theorem.

Theorem 3. Based on the proposed CA-CoMP scheme, the average harvested energy of a $M U$ per unit time, is given by

$$
\overline{\mathcal{Q}}=\overline{\mathcal{Q}}_{S}+\overline{\mathcal{Q}}_{I},
$$

where $\overline{\mathcal{Q}}_{S}$ and $\overline{\mathcal{Q}}_{I}$ are the average harvested energy from the intended signal and the interference, respectively, which are given by

$$
\begin{aligned}
\overline{\mathcal{Q}}_{S} & =T_{e}^{\mathrm{EH}} \zeta P_{t} G_{m} \int_{r_{1} \leq \cdots \leq r_{N}} \sum_{x_{i} \in \mathcal{C}} r_{i}^{-\alpha} f_{r}\left(r_{1}, \ldots, r_{N}\right) \mathrm{d} r_{1}, \ldots, r_{N}, \\
\overline{\mathcal{Q}}_{I} & =T_{e}^{\mathrm{EH}} \zeta P_{t} \sum_{G} \frac{2 \pi \lambda G p_{G}\left(\left(1-p_{a}\right) r_{m}^{2-\alpha}+p_{a} r_{N}^{2-\alpha}\right)}{\alpha-2},
\end{aligned}
$$

where $\zeta$ is the energy conversion efficiency, $r_{m}$ denotes the minimum distance from a $M U$ to the closest serving $B S$, and $T_{e}^{\mathrm{EH}}$ is the effective time for energy harvesting, which is given by

$$
T_{e}^{\mathrm{EH}}= \begin{cases}\max \left\{0,(1-\tau)\left(1-T_{b} \Xi_{b}-T_{c} \Xi_{c}\right)\right\} & \text { TS policy } \\ \max \left\{0,1-T_{b} \Xi_{b}-T_{c} \Xi_{c}\right\} & \text { PS policy. }\end{cases}
$$

Proof. See Appendix C.

\section{Numerical And Simulation Results}

We present the analytical and simulated results to validate the accuracy of our model and illustrate the performance of the CA-CoMP scheme. Unless otherwise stated, in our results we use the following parameters: $\lambda=1 / 2500, \alpha=3, N=2$, $n=3, v=30 \mathrm{~m} / \mathrm{s}, \tau=\rho=0.5, \zeta=0.7, T_{c}=0.8 \mathrm{~s}$,
$T_{b}=0.023 \mathrm{~s}, P_{t}=43 \mathrm{dBm}, r_{m}=1 \mathrm{~m}, \sigma_{\text {cov }}^{2}=-70 \mathrm{dBm}$ and $\sigma_{N}^{2}=-94 \mathrm{dBm}[8]$ [12].

Fig. 1 illustrates the effect of the area threshold on the coverage probability. In particular, Fig. 1 plots the coverage probability with respect to the SINR threshold for different area thresholds $\mathcal{A} \in\{0,2500,5000\} \mathrm{m}^{2}$, where the dash and solid curves present the TS and PS schemes of SWIPT technique, respectively. Firstly, it can be observed that TS scheme achieves a higher coverage probability than the PS scheme for different area thresholds $\mathcal{A}$. This was expected since, based on PS scheme, only a fraction $\rho$ of the received signal power is used for ID, resulting in a lower SINR at MUs. We can further observe that, by increasing the area threshold $\mathcal{A}$, the coverage probability drops. This is based on the fact that the density of BSs that satisfy the coverage area condition (i.e. the candidate BSs) is decreasing with larger area threshold, resulting in a longer distance from the MU to its serving BSs, thereby reducing the received signal strength.

Fig. 2 presents the impact of the area threshold $\mathcal{A}$ on the downlink spectral efficiency based on our proposed CA-CoMP scheme. Moreover, Fig. 2 plots the performance achieved by the conventional CoMP scheme [5] for comparison purpose. Since the traditional CoMP scheme does not depend on the area threshold, the achieved ASE remains constant. Clearly, it can be observed that our proposed CA-CoMP scheme outperforms the conventional scheme. It is interesting to note that at low area threshold values, the increasing of the threshold improves the network performance. However, by increasing the area threshold beyond a critical point, the network performance decreases. This observation is based on the fact that at low area threshold constants, the MUs experience less intra- and inter-cell handover process, while the MUs are still able to communicate with their serving BSs. In contrast, for the large area threshold values, the distances between a MU and its serving BSs increase, and thus the ASE significantly decreases. 
Another interesting observation is that, PS policy outperforms TS policy in terms of ASE. This can be explained by the fact that, by using the PS policy, more effective time can be allocated for decoding the received signal, resulting in the higher achieved data rate compared with the case with TS policy.

Finally, Fig. 3 shows the effect of the area threshold on the MUs' energy harvesting performance. In particular, Fig. 3 plots the average harvested energy of MUs versus different area threshold $\mathcal{A}$, where $v=\{10,20,30\} \mathrm{m} / \mathrm{s}$. Similar as Fig. 2, the energy harvesting performance achieved by the conventional CoMP scheme is illustrated for comparison purposes. Firstly, it can be observed that, compared with the CoMP scheme, our proposed CA-CoMP scheme is capable of enhancing the energy harvesting ability of MUs with different velocities, especially, for the MUs with high velocity. This was expected since the proposed scheme reduces the handover rate, thereby increasing the effective time for energy harvesting. Moreover, based on the CA-CoMP scheme, by increasing the area threshold, the MUs' average harvested energy is significantly improved. This is based on the fact that the density of candidate BSs decreases with a larger area threshold and, thus the handover rate of MUs decreases as well. Hence, MUs are able to utilise more time for harvesting energy. Similarly, it can be observed that, the MUs with a lower velocity are able to achieve a better energy harvesting performance because of the smaller handover rate.

\section{CONCLUSION}

In this paper, we investigated a novel CA-CoMP scheme, which was capable of significantly improving the performance of the SWIPT-enabled battery-operated MUs. In particular, based on the proposed cooperative scheme, MUs jointly communicated with several nearby serving BSs, of which coverage area satisfies the pre-defined area threshold. We investigated both inter- and intra-cell handover process experienced by MUs, where the closed-form expressions of the handover rates were derived. By leveraging stochastic geometry tools, analytical expressions for the coverage probability, the average spectral efficiency, as well as the average harvested energy were derived. Our analytical and simulated results have shown that, the proposed CA-CoMP effectively reduced the handover rate of MUs. Moreover, compared with the conventional CoMP scheme, our proposed CA-CoMP scheme achieved a higher average spectral efficiency and a higher average harvested energy of SWIPT-enabled MUs.

\section{APPENDIX A}

\section{PROOF OF THEOREM 1}

The proof follows a similar approach as in [8]. Since each MU jointly communicates with $N$ BSs, we first study the intra-cell handover with respect to the $k$-th closest serving $\mathrm{BS}$, i.e. $\mathrm{BS}_{k}$, where $k \in\{2, \cdots, N\}$. Without loss of generality, we consider the movement of a typical MU at origin, with trajectory from $(0,0)$ to $(1,0)$ along the $x$-axis. As shown in Fig. 4, the triangles denote the location of $\mathrm{BS}_{k}$ and $\mathrm{BS}_{k-1}$, the red circle $\omega_{k}$ denotes the location where the typical MU conducts the intra-cell handovers with respect to $\mathrm{BS}_{k}$, and $\theta_{k}$ is the angle of a beam boundary of $\mathrm{BS}_{k}$ with respect to the direction of the movement of the typical MU. Let $\Psi_{k}$ denote the point process of intra-cell handover with $\mathrm{BS}_{k}$. Hence, the average intra-cell handover rate with respect to $\mathrm{BS}_{k}$ is equivalent to the intensity of $\Psi_{k}$.

In order to calculate the intensity of $\Psi_{k}$, we start considering the case where there is at most one intra-cell handover corresponding to $\mathrm{BS}_{k}$, i.e. there are two beams of the same size for a BS. Thus, the event of intra-cell handover corresponding to $\mathrm{BS}_{k}$ occurs when the following two events occur simultaneously, i.e.

1) the point of the intra-cell handover lies in the $N$-th order Voronoi cell of the $N$ serving BSs, i.e. $\omega_{k} \in V_{\tilde{\Phi}}^{(N)}$, where $\mathrm{BS}_{k}$ is the $k$-th closest serving BSs of the typical MU.

2) the point of the intra-cell handover lies on the unit line connecting $(0,0)$ and $(1,0)$, i.e. $\omega_{k} \in[0,1]$.

Firstly, conditioning on $\theta_{k}$, the location of $\mathrm{BS}_{k}$ should be located on the strip between two lines passing through the origin and $(1,0)$. Moreover, the distance from the typical MU to the $\mathrm{BS}_{k}$ should not be less than the distance to $\mathrm{BS}_{k-1}$, i.e. $r_{k} \geq r_{k-1}$, where $r_{k}=\frac{\left|y_{k}\right|}{\left|\sin \theta_{k}\right|}$. Hence, by conditioning on both $\theta_{k}$ and $r_{k-1}$, the possible locations of $\mathrm{BS}_{k}$ are displayed as the shaded area in the Fig. 4, and the average number of intra-cell handovers in $[0,1]$ can be formulated as

$$
\begin{aligned}
& \mathbb{E}\left(\Psi_{k}[0,1] \mid \theta_{k}, r_{k-1}\right) \\
= & \tilde{\lambda} \int_{-\infty}^{-r_{k-1} \sin \theta_{k}} \mathrm{~d} y_{k} \int_{\frac{y_{k}}{\tan \theta_{k}}}^{1-\frac{y_{k}}{\tan \theta_{k}}} \exp \left(-\tilde{\lambda} \pi\left(\frac{y_{k}^{2}}{\sin ^{2} \theta_{k}}-r_{k-1}^{2}\right)\right) \mathrm{d} x_{k} \\
& +\tilde{\lambda} \int_{r_{k-1} \sin \theta_{k}}^{1-\frac{y_{k}}{\tan \theta_{k}}} \mathrm{~d} y_{k} \int_{\frac{y_{k}}{\tan \theta_{k}}}^{\infty} \exp \left(-\tilde{\lambda} \pi\left(\frac{y_{k}^{2}}{\sin ^{2} \theta_{k}}-r_{k-1}^{2}\right)\right) \mathrm{d} x_{k} \\
= & \exp \left(\pi \tilde{\lambda} r_{k-1}^{2}\right) \sqrt{\tilde{\lambda}}\left(\sin ^{2} \theta_{k}-\operatorname{erf}\left(\sqrt{\pi \tilde{\lambda}} r_{k-1}\right) \sin \theta_{k}\right),
\end{aligned}
$$

where $\operatorname{erf}(z)=\frac{2}{\pi} \int_{0}^{z} e^{-t^{2}} \mathrm{~d} t$ is the Gauss error function. Then, by averaging over $\theta_{k}$, which is uniformly distributed in $[0, \pi]$, we have

$\mathbb{E}\left(\Psi_{k}[0,1] \mid r_{k-1}\right)=2 e^{-\pi r_{k-1}^{2} \tilde{\lambda}} \sqrt{\tilde{\lambda}}\left(1-\operatorname{erf}\left(\sqrt{\pi \tilde{\lambda}} r_{k-1}\right)\right)\left(\pi^{-1}\right)$. Subsequently, by averaging over $r_{k-1}$, we can derive the linear intensity of $\Psi_{k}$ for the case of two beams as following

$$
\begin{aligned}
\mathbb{E}\left(\Psi_{k}[0,1]\right) & =\int_{0}^{\infty} \mathbb{E}\left(\Psi_{k}[0,1] \mid r_{k-1}\right) f_{k-1}\left(r_{k-1}\right) \mathrm{d} r_{k-1} \\
& =\frac{2 \sqrt{\tilde{\lambda}} \Gamma(k-0.5)}{\left.\pi^{3 / 2} \Gamma(k)\right)},
\end{aligned}
$$

where $f_{k}\left(r_{k}\right)=2(\pi \tilde{\lambda})^{n} r_{k}^{2 k-1} \frac{\exp \left(-\pi \tilde{\lambda} r_{k}^{2}\right)}{\Gamma(k)}$ is the PDF of distance from the typical MU to the $k$-th closest BS [11]. It should be noted that, the above formula also holds for the case $k=1$, where the proof is same as in [8] and hence is omitted. Then, for the case where each BS has $2^{n}$ beams, there are $2^{n-1}$ possibilities of intra-cell handovers corresponding to each serving BS. Hence, the linear intensity of intra-cell handovers 


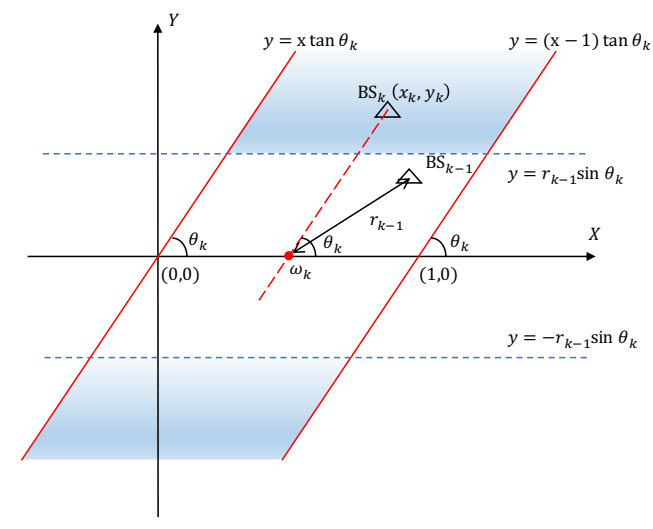

Fig. 4: Triangles: BSs' location, red circles: intra-cell handover location, dashed lines: beam boundaries, and shaded area: possible locations of $\mathrm{BS}_{k}$.

for the case where MU connects with $N$ serving BSs, is given by

$$
\Xi_{b}=2^{n-1} \sum_{k=1}^{N} \frac{2 \sqrt{\tilde{\lambda}} \Gamma(k-0.5)}{\pi^{3 / 2} \Gamma(k)} .
$$

Finally, by calculating the above summation as well as considering the velocity $v$ of the MU, the final expression for the intra-cell handover rate in Theorem 1 is proven.

\section{APPENDIX B}

ProOF OF THEOREM 2

The coverage probability can be re-written as,

$$
\begin{aligned}
& \mathcal{P}_{c}(\beta) \\
= & \mathbb{P}\left[\left|\sum_{x_{i} \in \mathcal{C}} G_{m}^{1 / 2} P_{t}^{1 / 2} r_{i}^{-\alpha / 2} h_{i}^{1 / 2}\right|^{2} \geq \beta\left(I+\sigma_{C}^{2}+\sigma_{N}^{2}\right)\right] \\
\stackrel{(a)}{=} & \mathbb{E}\left[\exp (-I s) \exp \left(-\left(\sigma_{C}^{2}+\sigma_{N}^{2}\right) s\right)\right] \\
= & \mathbb{E}_{r_{1}, \ldots, r_{N}}\left[\mathcal{L}_{I}(s) \exp \left(-\left(\sigma_{C}^{2}+\sigma_{N}^{2}\right) s\right)\right],
\end{aligned}
$$

where $I=\sum_{x_{i} \in \Phi \backslash \mathcal{C}} G P_{t} h_{i} r_{i}^{-\alpha}$ is the aggregate interference, $s=\beta\left(G_{m} P_{t}\left(r_{1}^{-\alpha}+\cdots+r_{N}^{-\alpha}\right)\right)^{-1}$ and $(a)$ is from the fact that given $h_{i} \sim \exp (1),\left|\sum_{x_{i} \in \mathcal{C}} G_{m}^{1 / 2} P_{t}^{1 / 2} r_{i}^{-\alpha / 2} h_{i}^{1 / 2}\right|^{2}$ is an exponential random variable with mean $\left(\sum_{x_{i} \in \mathcal{C}} G_{m} P_{t} r_{i}^{-\alpha}\right)^{-1}$. Then, the Laplace transform of the interference function can be derived as

$$
\begin{aligned}
& \mathcal{L}_{I}(s)= \mathbb{E}\left[\exp \left(\sum_{x_{i} \in \Phi \backslash \mathcal{C}}-s G P_{t} h_{i} r_{i}^{-\alpha}\right)\right] \\
&= \mathbb{E}\left[\prod_{x_{i} \in \Phi \backslash \mathcal{C}} \exp \left(\frac{1}{1+s P_{t} G r_{i}^{-\alpha}}\right)\right] \\
& \stackrel{(b)}{=} \exp \left(2 \pi \lambda\left(1-p_{a}\right) \int_{0}^{r_{N}}(z(s, r)-1) r \mathrm{~d} r\right) \times \\
& \\
& \quad \exp \left(2 \pi \lambda \int_{r_{N}}^{\infty}(z(s, r)-1) r \mathrm{~d} r\right),
\end{aligned}
$$

where $(b)$ follows from the probability generating function of a PPP and $z(s, r)=\sum_{G} p_{G} /\left(1+s P_{t} G r^{-\alpha}\right)$. Then, by using $[13,3.194 .5]$ and by evaluating the expectation over distance $r_{1}, \ldots, r_{N}$, the final results in Theorem 2 are derived.

\section{APPENDIX C \\ PROOF OF THEOREM 3}

The average harvested energy per unit time can be evaluated by averaging the instantaneous harvested energy over the random channel and path loss components, i.e.

$$
\overline{\mathcal{Q}}=T_{e}^{\mathrm{EH}} \mathbb{E}\left[\sum_{x_{i} \in \mathcal{C}} P_{t} G_{m} h_{i} r_{i}^{-\alpha}+\sum_{x_{i} \in \Phi \backslash \mathcal{C}} P_{t} G h_{i} r_{i}^{-\alpha}\right],
$$

where the first and the second term represent the average harvested energy from the intended and interfering signals, respectively. The average harvested energy from the intended signals, $\mathcal{Q}_{s}$, can be evaluated as following

$$
\begin{aligned}
\overline{\mathcal{Q}}_{S} & =T_{e}^{\mathrm{EH}} \mathbb{E}\left[P_{t} G_{m}\left(r_{1}^{-\alpha}+\cdots+r_{N}^{-\alpha}\right)\right] \\
& =\int_{r_{m} \leq r_{1} \leq \cdots \leq r_{N}} P_{t} G_{m}\left(r_{1}^{-\alpha}+\cdots+r_{N}^{-\alpha}\right) f_{r}\left(r_{1}, \ldots, r_{N}\right) \mathrm{d} r_{1}, \ldots, r_{N} .
\end{aligned}
$$

The average harvested energy from interfering signals is given by

$$
\begin{aligned}
& \overline{\mathcal{Q}}_{I}=\mathbb{E}\left[\sum_{x_{i} \in \Phi \backslash \mathcal{C}} P_{t} G h_{i} r_{i}^{-\alpha}\right] \\
= & \sum_{G} P_{t} G p_{G}\left(\int_{r_{m}}^{r_{N}} 2 \pi \lambda\left(1-p_{a}\right) r^{1-\alpha} \mathrm{d} r+\int_{r_{N}}^{\infty} 2 \pi \lambda r^{1-\alpha} \mathrm{d} r\right) .
\end{aligned}
$$

Finally, by evaluating the above integrals, the final results in Theorem 3 is proven.

\section{REFERENCES}

[1] I. Krikidis, S. Timotheou, S. Nikolaou, G. Zheng, D. W. K. Ng, and R. Schober, "Simultaneous wireless information and power transfer in modern communication systems," IEEE Commun. Mag., vol. 52, no. 11, pp. 104-110, Nov. 2014.

[2] R. Zhang and C. K. Ho, "MIMO broadcasting for simultaneous wireless information and power transfer," IEEE Trans. Wireless Commun., vol. 12, no. 5, pp. 1989-2001, May 2013.

[3] M. Di Renzo and W. Lu, "System-level analysis and optimization of cellular networks with simultaneous wireless information and power transfer: Stochastic geometry modeling," IEEE Trans. Veh. Technol., vol. 66, no. 3, pp. 2251-2275, Mar. 2017.

[4] T. Tu Lam, M. Di Renzo, and J. P. Coon, "System-level analysis of SWIPT MIMO cellular networks," IEEE Commun. Lett., vol. 20, no. 10, pp. 2011-2014, Oct. 2016.

[5] W. Bao and B. Liang, "Optimizing cluster size through handoff analysis in user-centric cooperative wireless networks," IEEE Trans. Wireless Commun., vol. 17, no. 2, pp. 766-778, Feb. 2018.

[6] W. Sun, L. Wang, J. Liu, N. Kato, and Y. Zhang, "Movement aware CoMP handover in heterogeneous ultra-dense networks," IEEE Trans. Commun., vol. 69, no. 1, pp. 340-352, Jan. 2021.

[7] J. Tang, A. Shojaeifard, D. K. C. So, K. Wong, and N. Zhao, "Energy efficiency optimization for CoMP-SWIPT heterogeneous networks," IEEE Trans. Commun., vol. 66, no. 12, pp. 6368-6383, Dec. 2018.

[8] S. S. Kalamkar, F. M. Abinader, F. Baccelli, A. S. M. Fani, and L. G. U. Garcia, "Stochastic geometry-based modeling and analysis of beam management in 5G," in IEEE Global Commun. Conf. (GLOBECOM), Taipei, Dec. 2020, pp. 1-6.

[9] M. Haenggi, Stochastic Geometry for Wireless Networks. Cambridge University Press, 2012.

[10] X. Lin, R. K. Ganti, P. J. Fleming, and J. G. Andrews, "Towards understanding the fundamentals of mobility in cellular networks," IEEE Trans. Wireless Commun., vol. 12, no. 4, pp. 1686-1698, Apr. 2013.

[11] D. Moltchanov, "Distance distributions in random networks," Ad Hoc Netw., vol. 10, no. 6, pp. 1146-1166, Aug. 2012.

[12] E. Demarchou, C. Psomas, and I. Krikidis, "Mobility management in ultra-dense networks: Handover skipping techniques," IEEE Access, vol. 6, pp. 11921-11930, Feb. 2018.

[13] I. S. Gradshteyn and I. M. Ryzhik, Table of Integrals, Series, and Products. New York, NY, USA: Elsevier, 2007. 